\title{
Prognostic and predictive value of common mutations for treatment response and survival in patients with metastatic colorectal cancer
}

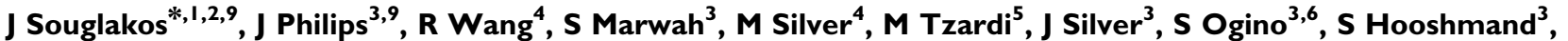 E Kwak $^{7,8}$, E Freed ${ }^{3}$, JA Meyerhardt ${ }^{3,8}$, Z Saridaki ${ }^{2}$, V Georgoulias ${ }^{1,2}$, D Finkelstein ${ }^{4}$, CS Fuchs ${ }^{3,8}$, MH Kulke,8 and RA Shivdasani*,3,8}

'Department of Medical Oncology, University Hospital, Heraklion, Crete, Greece; ${ }^{2}$ Laboratory of Tumor Biology, University of Crete, Heraklion, Crete, Greece; ${ }^{3}$ Department of Medical Oncology, Dana-Farber Cancer Institute, Boston, Massachusetts, USA; ${ }^{4}$ Department of Biostatistics, Massachusetts General Hospital, Boston, Massachusetts, USA; ${ }^{5}$ Department of Pathology, Laboratory of Pathology, University Hospital, Heraklion, Crete, Greece; ${ }^{6}$ Department of Pathology, Brigham and Women's Hospital, Boston, Massachusetts, USA; ${ }^{7}$ Department of Medicine, Massachusetts General Hospital, Boston, Massachusetts, USA; ${ }^{8}$ Department of Medicine, Harvard Medical School, Boston, Massachusetts, USA

BACKGROUND: We address the prognostic and predictive value of KRAS, PIK3CA and BRAF mutations for clinical outcomes in response to active agents in the treatment of metastatic colorectal cancer (mCRC).

METHODS: We determined KRAS, BRAF and PIK3CA mutations in tumours from 168 patients treated for mCRC at two institutions. All patients received 5-FU-based first-line chemotherapy and treatment outcome was analysed retrospectively.

RESULTS: KRAS, BRAF and PIK3CA mutations were present in 62 (37\%), 13 (8\%) and 26 (I 5\%) cases, respectively. Multivariate analysis uncovered BRAF mutation as an independent prognostic factor for decreased survival (hazard ratio (HR) 4.0, 95\% confidence interval (CI) 2.1 -7.6). In addition, patients with BRAF-mutant tumours had significantly lower progression-free survival (PFS: HR 4.0, 95\% $\mathrm{Cl} 2.2-7.4)$ than those whose tumors that carried wild-type BRAF. Among 92 patients treated using chemotherapy and cetuximab as salvage therapy, KRAS mutation was associated with lack of response $(P=0.002)$ and shorter PFS $(P=0.09) . B R A F(P=0.0005)$ and PIK3CA $(P=0.01)$ mutations also predicted reduced PFS in response to cetuximab salvage therapy.

CONCLUSIONS: These results underscore the potential of mutational profiling to identify CRCs with different natural histories or treatment responses. The adverse significance of BRAF mutation should inform patient selection and stratification in clinical trials. British Journal of Cancer (2009) I 0 I, 465-472. doi:I0.I038/sj.bjc.6605 I64 www.bjcancer.com

Published online 14 July 2009

(C) 2009 Cancer Research UK

Keywords: KRAS; PIK3CA; BRAF; mutation; prediction; metastatic CRC

Combinatorial and sequential administration of 5-fluorouracil (5-FU), oxaliplatin and irinotecan has substantially increased the overall survival (OS) in metastatic colorectal cancer (mCRC), although treatment goals remain primarily palliative (Grothey et al, 2004). Monoclonal antibodies (mAb) bevacizumab, against the vascular endothelial growth factor (VEGF) receptor, and cetuximab or panitumumab, against the epidermal growth factor receptor (EGFR), expand treatment options and further improve progression-free (PFS) and OS (Cunningham et al, 2004; Hurwitz et al, 2004; Van et al, 2007).

The genetic underpinnings of colorectal cancer (CRC) are especially well characterized (Vogelstein and Kinzler, 2004) and

\footnotetext{
*Correspondence: Dr J Souglakos, University Hospital of Heraklion, Voutes and Stavrakia, PO BOX 1352, Heraklion, Crete 71।10, Greece. E-mail: georgsec@med.uoc.gr or Dr R Shivdasani, Dana-Farber Cancer Institute, 44 Binney Street, Boston, MA 02115, USA;

E-mail: ramesh_shivdasani@dfci.harvard.edu

${ }^{9}$ These authors contributed equally to this work

Received 2 February 2009; revised 29 May 2009; accepted 5 June 2009; published online 14 July 2009
}

include common somatic mutations in the APC, TP53 and KRAS genes, followed in frequency by PIK3CA and BRAF mutations (Wood et al, 2007). Activating mutations in the KRAS, BRAF and PIK3CA oncogenes deregulate growth-factor pathways, stimulate cell proliferation and promote metastasis (Huang et al, 2007; Schubbert et al, 2007). Presence of KRAS mutations in primary CRC portends a poor response to monotherapy and combination therapy using anti-EGFR mAB cetuximab or panitumumab (Amado et al, 2008; Karapetis et al, 2008; Lievre et al, 2008). In one study that included few patients with stage IV disease and $B R A F$ mutations, the latter were associated with limited survival in patients with microsatellite-stable (MSS) disease (Samowitz et al, 2005); mutations in exons 9 or 20 of the PIK3CA gene are associated with lower relapse-free survival in patients with stage II or III CRC (Kato et al, 2007). These data notwithstanding, there is limited understanding of the extent to which common KRAS, $B R A F$ and PIK3CA mutations influence clinical outcomes in patients with mCRC treated with chemotherapy and/or biological therapy. Treatments are selected largely without regard for tumour mutation profiles, although recent evidence linking KRAS mutation with poor response to EGFR mAb (Amado et al, 2008; 
Karapetis et al, 2008; Lievre et al, 2008) now influences decisions in the clinic.

We postulated that mutations in some of these oncogenes would correlate with overall prognosis and with treatment outcomes in patients receiving different regimens. To test these hypotheses, we first evaluated the prognostic significance of $K R A S, B R A F$ and PIK3CA mutations in a cohort of patients with mCRC, treated using 5FU-based chemotherapy. Using a uniform catalogue of retrospective but detailed clinical information, we then tested the predictive value of these mutations on patient outcomes after treatment using the most common therapeutic regimens.

\section{MATERIALS AND METHODS}

\section{Patients and study design}

Tumour samples were collected from patients with histologically confirmed mCRC, treated at two centers, Dana-Farber/Partners Cancer Care (Boston, MA, USA) and University Hospital of Heraklion (Heraklion, Crete, Greece), between January 2004 and March 2007. All patients who gave informed consent and whose colectomy samples were available for molecular analysis were included in the analysis, without further selection. Failure to participate in the tumour registry was unusual, and the cohort essentially represents consecutive patients with mCRC who were treated at the two academic centres and gave informed consent for molecular analysis of DNA from the primary tumour. Institutional ethics committees approved the study.

First-line and salvage regimens, selected at the discretion of the treating physician, followed common practices in mCRC (summarized in Table 1). Patients were evaluated at baseline and after every four cycles of therapy, with occasional variation, as clinically indicated, during the treatment. Disease status was coded, without the knowledge of the mutational profile, after retrospective review of physician notes and reports of staging radiographs, including tumour measurements; efficacy was assessed on an intent-to-treat basis. Disease progression was defined as the appearance of any new lesion or a greater than $20 \%$ increase in the sum of the longest diameter (LD) of target lesions, extracted from radiology reports, and taking as reference the lowest value recorded in the treatment period. Response toward cetuximab was defined as the reduction, by at least $30 \%$, in the sum of the LD of target lesions compared with the baseline sum.

Table I Treatment regimens used in this retrospective study

\begin{tabular}{lcr}
\hline First-line regimens & N (out of 168) & \% \\
\hline FOLFOX+bevacizumab & 76 & 45 \\
FOLFIRI+bevacizumab & 19 & 11 \\
5FU+bevacizumab & 2 & 1 \\
FOLFOX+cetuximab & 8 & 5 \\
FOLFOXIRI & 17 & 10 \\
FOLFIRI & 25 & 15 \\
FOLFOX & 16 & 10 \\
5FU only & 5 & 3 \\
Oxaliplatin-based treatment (first line) & 100 & 60 \\
Irinotecan-based treatment (first line) & 44 & 26 \\
Bevacizumab+chemotherapy (first line) & 97 & 58 \\
Oxaliplatin-based treatment (any line) & 144 & 86 \\
Irinotecan-based treatment (any line) & 121 & 72 \\
Bevacizumab+chemotherapy (any line) & 131 & 78 \\
Cetuximab+chemotherapy (any line) & 100 & 60 \\
Patients treated with all three chemotherapy drugs & 106 & 63 \\
Patients treated with all five active agents & 87 & 52 \\
\hline
\end{tabular}

FOLFIRI = Folinic acid, 5FU, irinotecan; FOLFOX = Folinic acid, 5FU, oxaliplatin $\mathrm{FOLFOXIRI}=$ Folinic acid, 5FU, oxaliplatin, irinotecan

\section{Specimen Characteristics and Assay Methods}

Formalin-fixed, paraffin-embedded tumour sections were reviewed by pathologists to confirm the diagnosis and define tumourenriched areas for dissection. From each paraffin block of representative tumour areas, we stained 10 serial sections of $8 \mu \mathrm{m}$ thickness with nuclear fast red (Sigma-Aldrich, St Louis, MO, USA) and isolated malignant cells by scrape dissection under a binocular microscope. Macrodissected cells were lysed in buffer containing Proteinase K (Puregene, Gentra Systems, Minneapolis, $\mathrm{MN}$, USA) at $60^{\circ} \mathrm{C}$ for $72 \mathrm{~h}$, followed by DNA extraction according to the manufacturer's protocol.

Mutations were detected in the Dana-Farber sample set by Sequenom mass-spectrometric genotyping (Thomas et al, 2007) after whole-genome amplification by PCR, and in the Heraklion samples by Sanger sequencing after PCR amplification of KRAS exon 2, BRAF exon 15, and PIK3CA exons 9 and 20. PCR conditions and the primers used to amplify specific exons are listed in Supplementary Table 1. Results from the two different methods for detecting mutations were cross-confirmed on the other platform, along with positive and negative controls.

\section{Statistical analysis}

Associations between treatment response and mutation status or baseline characteristics were assessed using the Fisher's exact test for dichotomous variables, or logistic regression for continuous variables. PFS was measured from the last radiographic study before the initiation of first-line therapy to the first radiographic documentation of disease progression or death, and OS was calculated from the date of diagnosis of metastatic disease to death due to any cause. For cetuximab treatment, PFS was calculated from the date of documented progression after the previous treatment to the next documentation of disease progression or death. Kaplan-Meier curves were used to describe the proportion of patients who remained free of events over the follow-up period. Associations between prognostic factors and PFS or OS were examined using Cox proportional hazards regression models; we report hazard ratio (HR) estimates and their 95\% confidence intervals $(\mathrm{CI})$.

We used Cox regression models with interaction terms to assess whether mutational effects varied across treatment subgroups. For each mutation, three hypotheses were tested: (1) whether effect on first-line PFS varied according to regimen (oxaliplatin based, irinotecan based or both); (2) whether the effect on first-line PFS varied according to inclusion or exclusion of bevacizumab; and (3) whether the effect on PFS for cetuximab varied according to the line of treatment (first $v s \geqslant$ third). All reported $P$-values are twosided and not adjusted for multiple testing.

\section{RESULTS}

\section{Patient and tumour characteristics, treatments and clinical outcomes}

We determined with confidence the mutational status for KRAS exon 2, BRAF exon 15, and PIK3CA exons 9 and 20 in 168 consecutive patients with $\mathrm{mCRC}$, for whom a representative sample from the primary tumour was available for molecular analysis of DNA. Disease characteristics were typical for mCRC in North America and Europe (Table 2), and patients were treated according to prevailing practice patterns (Table 1). KRAS mutations were detected in $62(37 \%), B R A F$ mutations in 13 $(8 \%)$ and PIK3CA mutations in $26(15 \%, 18$ in exon 9 and 8 in exon 20) primary tumours, respectively. Seven tumours carried both KRAS and PIK3CA mutations, whereas BRAF mutations did not co-occur with the others; these frequencies closely match those in published reports (Wood et al, 2007) and were similar between the 
Table 2 Characteristics of enrolled patients and univariate analysis for survival

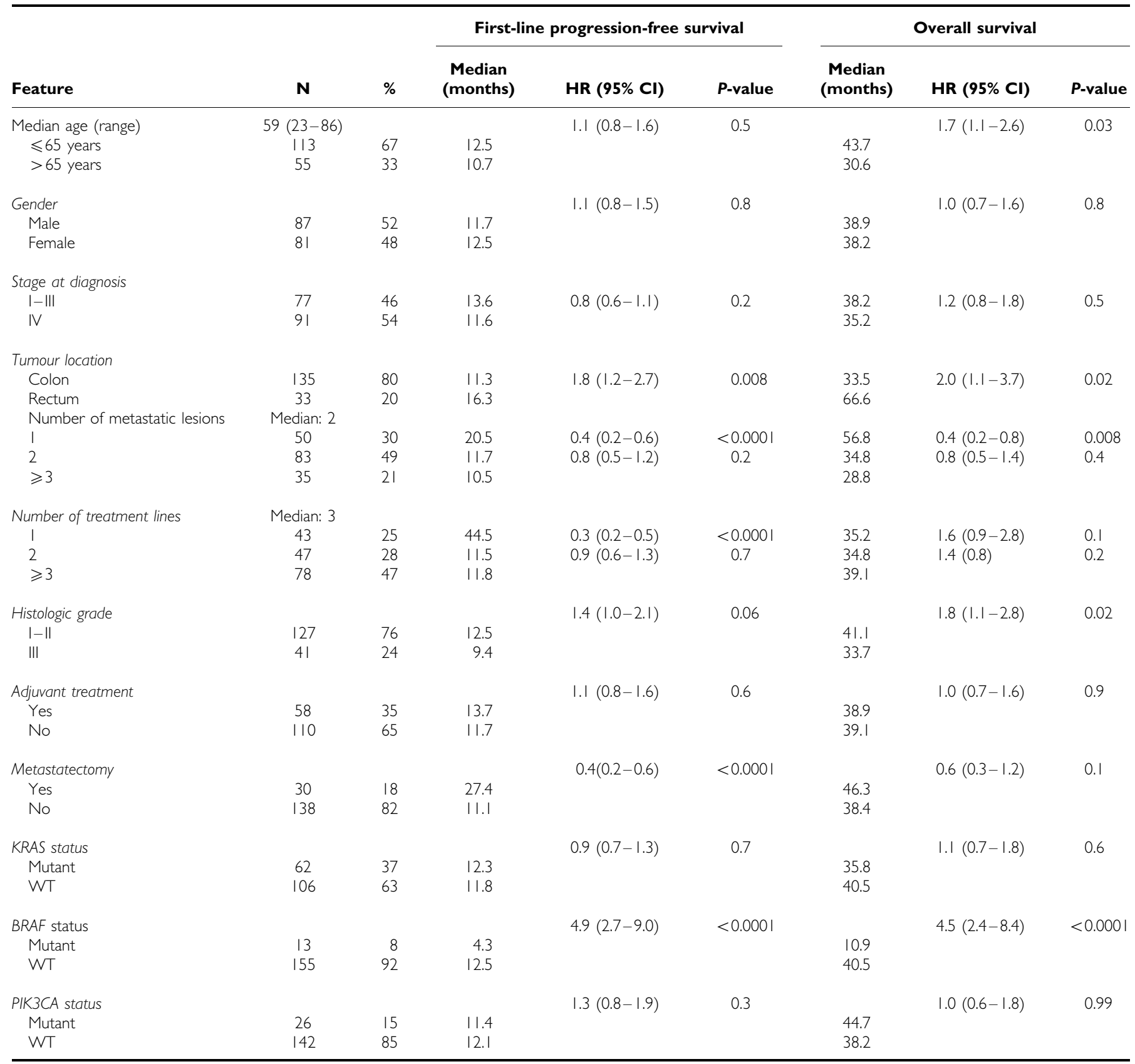

$\mathrm{HR}=$ hazard ratio; $\mathrm{WT}=$ wild type.

US and Greek cohorts (Supplementary Table 2). Mutations in any gene did not correlate significantly with patient gender or age, stage at diagnosis, or histological grade (all $P$-values $>0.05$ ). As patient characteristics, mutation frequencies and treatment regimens were all typical for CRC, the results of our analysis should serve as a useful guide for clinical practice.

The median time from initial diagnosis to diagnosis of metastatic disease was 18.4 months (95\% CI 14.4-20.4) for patients with stage I-III disease at presentation and 0.6 months (95\% CI $0.1-1.4)$ for patients classified with stage IV disease. The median time from the last radiological assessment to the initiation of first-line chemotherapy was 0.6 months (95\% CI $0.4-0.8$ ), whereas median duration from the diagnosis of metastatic disease was 1.2 months (95\% CI $0.9-1.3)$.

OS was similar between the two participating centres (median 38.9 months, 95\% CI 33.4-46.3 months) and higher than other published values (Grothey et al, 2004; Hurwitz et al, 2004). Considering that patients were not selected on the basis of performance status or other clinical criteria, one possibility for improved outcomes may be that after initial response to systemic treatment, 30 out of 50 patients with solitary metastases (representing $18 \%$ of the total cohort) had metastases resected. Response to particular agents was, however, monitored in all patients and we examined the role of metastatectomy as a variable. Univariate analysis (Table 2) showed significant associations of OS with age $>65$ years $(P=0.03)$, undifferentiated (grade 3$)$ primary tumours $(P=0.02)$, colonic vs rectal location $(P=0.02)$, solitary metastasis $(P=0.008)$ and $B R A F$ mutations $(P<0.0001$, Figure 1$)$. When all factors significant in univariate analysis were subjected to multivariate analysis (Table 3 ), only $B R A F$ mutation remained as an independent prognostic factor for reduced OS (HR 4.0, 95\% CI $2.1-8.0$ ). 

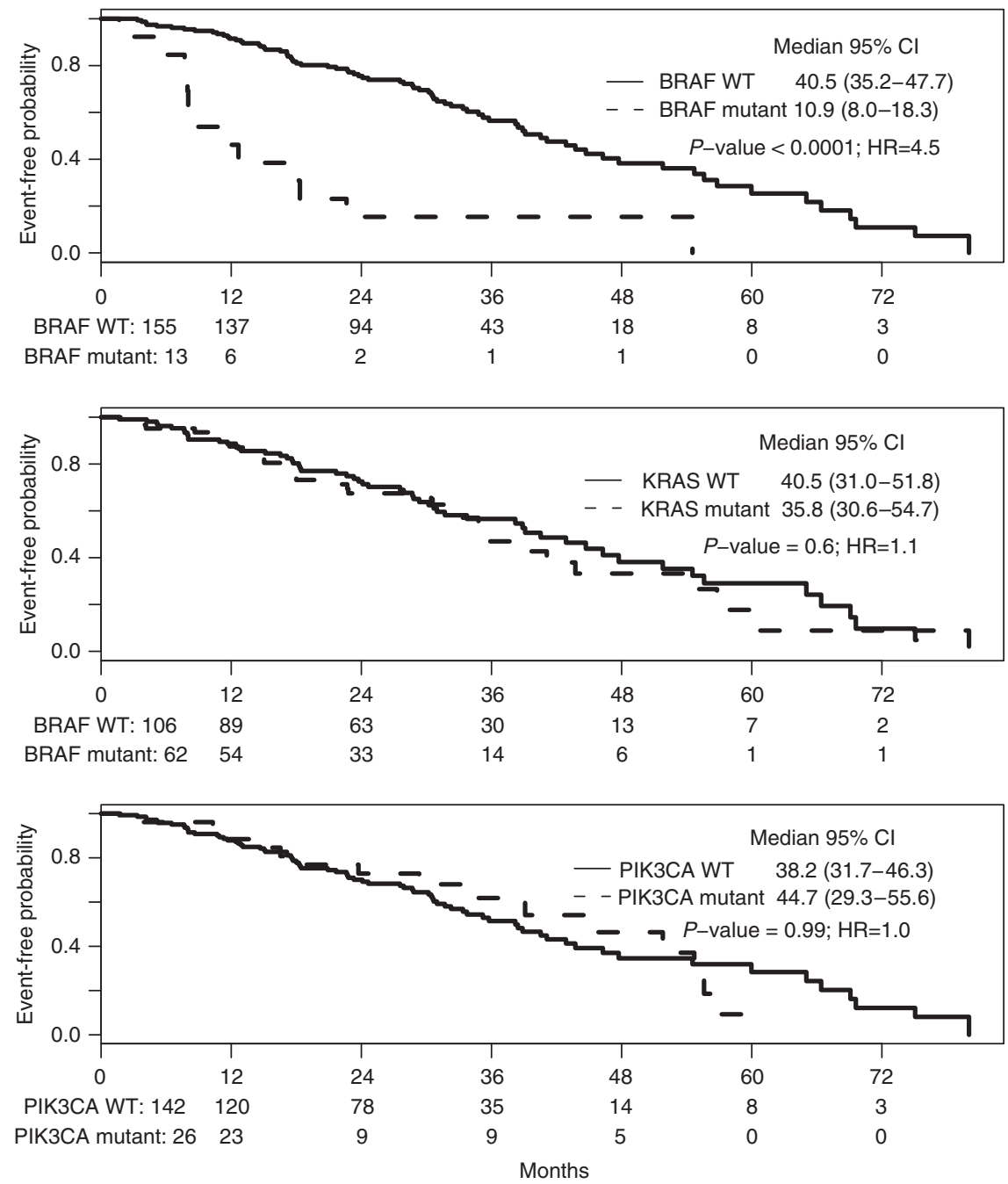

Figure I Overall survival after the diagnosis of metastatic disease, analysed by mutation status.

Table 3 Results of multivariate analysis for progression-free and overall survival

\begin{tabular}{|c|c|c|c|}
\hline & Hazard ratio & $95 \% \mathrm{Cl}$ & $P$-value \\
\hline \multicolumn{4}{|l|}{ Progression-free sunvival } \\
\hline BRAF (mutant vs WT*) & 4.0 & $(2.2,7.4)$ & $<0.000$ \\
\hline Age ( $>65$ vs $\leqslant 65$ years $)$ & 1.4 & $(1.0,2.0)$ & 0.06 \\
\hline Tumour grade (3 vs $1-2)$ & 1.5 & $(1.0,2.2)$ & 0.06 \\
\hline Metastatectomy (yes vs no) & 0.6 & $(0.4,1.1)$ & 0.08 \\
\hline Tumour location (colon vs rectum) & 1.6 & $(1.0,2.4)$ & 0.05 \\
\hline Number of treatment lines (I vs >3) & 0.3 & $(0.2,0.5)$ & $<0.0001$ \\
\hline Number of treatment lines ( 2 vs $>3$ ) & 0.9 & $(0.6,1.3)$ & 0.5 \\
\hline \multicolumn{4}{|l|}{ Overall sunvival } \\
\hline BRAF (mutant vs WT) & 4.1 & $(2.1,8.0)$ & $<0.000$ \\
\hline Age ( $>65$ vs $\leqslant 65$ years $)$ & 1.5 & $(1.0,2.4)$ & 0.07 \\
\hline Tumour grade (3 vs I-2) & 1.3 & $(0.8,2.1)$ & 0.3 \\
\hline Metastatectomy (yes vs no) & 0.6 & $(0.3,1.3)$ & 0.2 \\
\hline Stage at diagnosis (I-III vs IV) & 1.3 & $(0.8,2.0)$ & 0.2 \\
\hline Tumor location (colon vs rectum) & 1.8 & $(0.9,3.3)$ & 0.08 \\
\hline Number of lines of treatment ( 1 vs $\geqslant 3$ ) & 1.8 & $(0.98,3.3)$ & 0.06 \\
\hline Number of lines of treatment ( 2 vs $\geqslant 3$ ) & 1.4 & $(0.8,2.3)$ & 0.3 \\
\hline
\end{tabular}

$\mathrm{Cl}=$ confidence interval; $\mathrm{WT}=$ wild type.
Impact of mutation status on the outcome of first-line chemotherapy

Combination chemotherapy was administered in the first line in 161 patients (96\%), with or without mAb supplementation (Table 1$) ; 5$ patients $(3 \%)$ received 5 -FU as the only cytotoxic agent. One hundred patients $(60 \%)$ were treated with the folinic acid-fluororuracil-oxaliplatin (FOLFOX) regimen, with or without the addition of a $\mathrm{mAb}$, in the first line and 97 patients $(58 \%)$ received bevacizumab in addition to fluoropyrimidine-based chemotherapy; a smaller fraction, $24 \%$ (44 patients) was treated with irinotecan in the first line. Seventeen patients $(10 \%)$ received both oxaliplatin and irinotecan (Table 1). In all, 106 patients (63\%) received all three active chemotherapy drugs and 87 patients (52\%) received all five active agents at some point in their disease course.

KRAS or PIK3CA mutations did not influence PFS after first-line therapy (Figure 2). In contrast, patients with $B R A F$-mutant tumours had significantly lower PFS (median 4.3 vs 12.5 months; $P<0.0001)$ compared with patients whose primary tumours carried only wild-type (WT) $B R A F$ alleles (Figure 2). The association between BRAF mutation and reduced PFS (HR 4.9, 95\% CI 2.7-9.0) after first-line therapy (Table 3) was similar for patients receiving oxaliplatin-, irinotecan- or both (interaction test 

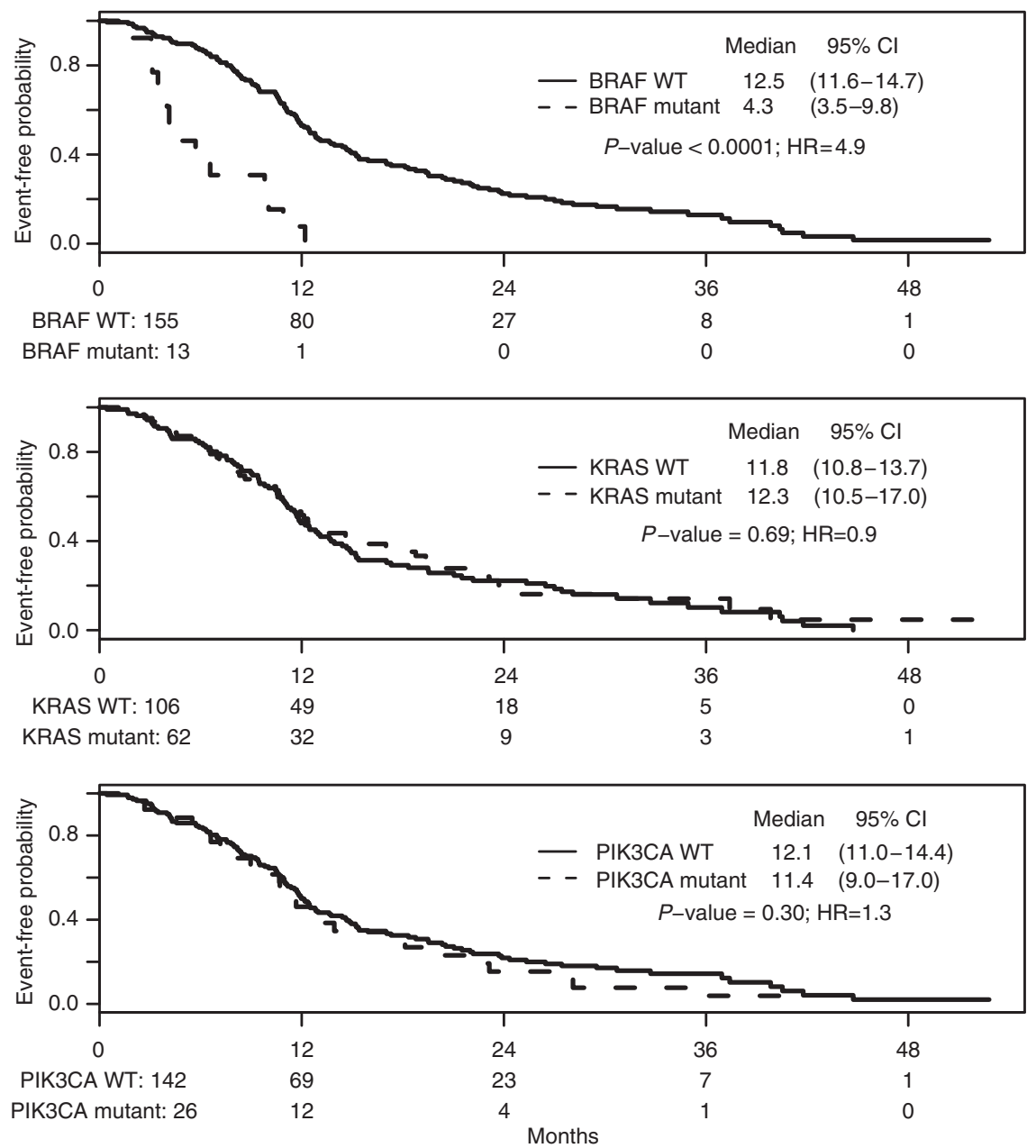

Figure 2 Progression-free survival after first-line chemotherapy, analysed by mutation status.

$P=0.7$ ), and similar for patients receiving a regimen with or without bevacizumab (interaction test $P=0.8$ ). Specifically, $B R A F$ mutation predicted more rapid disease progression in patients treated with first-line oxaliplatin- (HR 6.4,95\% CI 2.6-15.6), irinotecan- (HR 4.1, 95\% CI 1.5-11.3), or oxaliplatin and irinotecan (HR 7.9, 95\% CI 1.3-48.2), as well as bevacizumabcontaining (HR 5.1, 95\% CI 2.4-11.1) regimens (Supplementary Table 3). Thus, KRAS and PIK3CA mutations did not show predictive value with respect to cytotoxic agents and our observations single out $B R A F$ mutation in the primary tumour as an adverse factor, independent of the first-line treatment.

\section{Impact of mutation status on the outcome of salvage cetuximab therapy}

One hundred patients were treated with cetuximab, 8 in the first line, 37 in the second and 55 in the third or higher, always in combination with chemotherapy. All patients receiving cetuximab in the second or subsequent line were refractory to previous regimens. Restricting analysis to the 92 patients who received cetuximab combinations as salvage therapy, none of the 32 patients with a KRAS-mutant tumour responded to treatment, whereas objective responses occurred in 14 of 60 (23\%) patients whose tumors harboured only WT KRAS alleles $(P=0.002)$. This striking difference confirms the results from prospective trials of cetuximab or panitumumab monotherapy (Amado et al, 2008; Karapetis et al, 2008; Lievre et al, 2008) and validates our approach to identify associations to guide the treatment of mCRC. It should be noted that none of the 9 patients with a $B R A F$-mutant tumour responded to cetuximab, whereas objective responses occurred in 14 of $83(17 \%)$ patients whose tumours carried WT BRAF alleles. Presence of PIK3CA mutations showed no correlation with objective tumour responses to cetuximab. PFS was significantly lower among patients whose tumours carried mutations in BRAF (2.0 vs 3.9 months, HR 3.6, 95\% CI 1.8-7.4), PIK3CA (2.5 vs 3.9 months, HR $2.1,95 \%$ CI $1.2-3.9)$ or any of the three genes $(2.5 \mathrm{vs}$ 6.4 months, HR 2.1, 95\% CI 1.3-3.2), and marginally lower in patients with KRAS-mutant tumours (2.5 vs 4.8 months, HR 1.5, 95\% CI 0.9-2.3), compared with patients whose primary tumors were wild type at each locus (Figure 3, Supplementary Table 3). Importantly, patients whose tumour had BRAF or PIK3CA mutation had shorter PFS regardless of whether cetuximab was administered in the second, or third and higher lines (interaction tests $P=0.5$ and 0.6 , respectively). Thus, the lack of EGFR mAb response observed in the setting of mutant KRAS (Schubbert et al, 2007; Amado et al, 2008) extends to other common mutations that deregulate cellular signalling pathways, especially BRAF and probably even PIK3CA. Lower PFS did not translate into differences in OS between patients with WT or mutant KRAS and PIK3CA alleles in the primary tumour.

\section{DISCUSSION}

Among the many genetic alterations in CRC, particular point mutations in three oncogenes, KRAS, PIK3CA and BRAF, occur at 

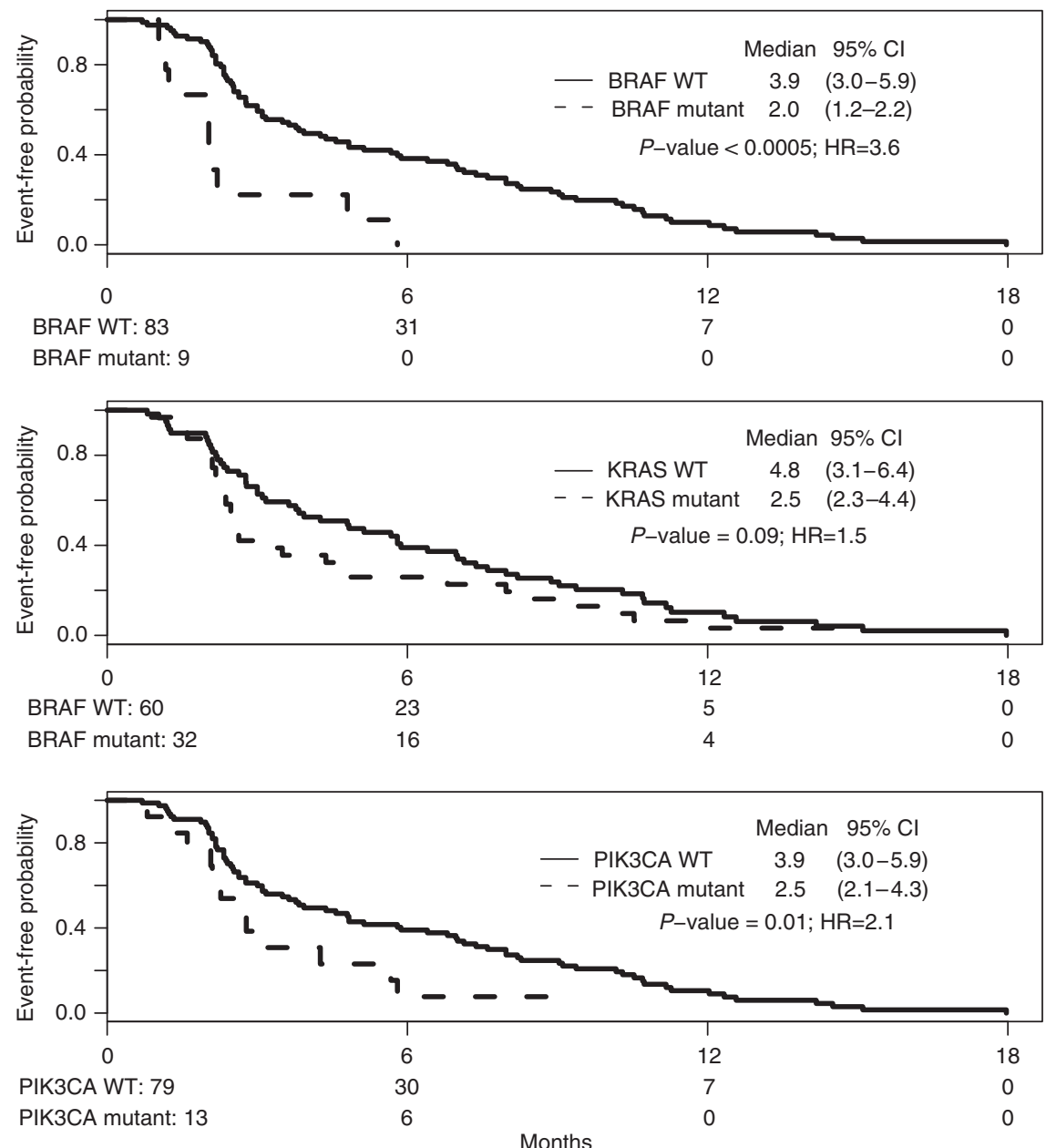

Figure 3 Progression-free survival after salvage cetuximab therapy in 92 patients, analysed by mutation status.

sufficiently high frequencies to be implicated in disease pathogenesis and as targets for molecular therapy (Wood et al, 2007). Each of these targets is the subject of intense drug development efforts. Appreciation of the predictive significance of underlying oncogene mutations will aid in design of clinical trials of new agents, administered either alone or in combination with other drugs, and in interpreting results from new and old trials. Moreover, current treatment approaches toward mCRC rarely consider biological properties associated with individual mutations, mainly because there is a paucity of information about the influence of specific mutations on natural history or the response to particular treatments. We report the results of retrospective investigation designed to help define the role of tumour mutation profiles in the management of patients with mCRC. Even though treatment choices and staging studies were not dictated by a strict clinical trial, the results are robust and relate well to current treatment paradigms.

A principal finding was that $B R A F$ mutation the primary tumour marks patients who carry an especially poor prognosis, regardless of specific treatment regimen. Compared with patients with $B R A F^{\mathrm{WT}}$ tumours, those with $B R A F^{M U T}$ tumours have significantly higher likelihood of disease progression $(P<0.0001)$ or death $(P<0.0001)$ with any current regimen (Table 3$)$; the BRAF V600E mutation predicted independently for early relapse on first-line therapy (HR 4.0, 95\% CI 2.2-7.4) and death (HR 4.0, 95\% CI $2.1-8.0$ ). These data warrant confirmation and should provoke thoughtful consideration of the risks and benefits of administering 5-FU, oxaliplatin or irinotecan in such patients, who should probably also be considered as a distinct group by stratification in future clinical trials. The poorer PFS with first-line therapy suggests that BRAF mutation could be prognostic, predictive or both. As this association was highly significant for patients receiving oxaliplatin, irinotecan or both, as well as for patients receiving bevacizumab or not, the conservative interpretation is that $B R A F$ mutation is a prognostic rather than a predictive marker.

Our observations have interesting implications for pathogenic mechanisms and disease subgroups. Activating mutations in both $K R A S$ and BRAF stimulate the MEK or extracellular signalregulated kinase-microtubule-associated protein (ERK-MAP) kinase cascade (Vogelstein and Kinzler, 2004). As the two mutations tend to be mutually exclusive (Rajagopalan et al, 2002; Yuen et al, 2002), KRAS is inferred to signal principally through $B R A F$ in colon cancer cells, leading to an assumption that tumours with KRAS or BRAF mutations may be equally vulnerable to inhibition of $B R A F$. Our finding that the natural history and treatment response of $B R A F$-mutant tumours differ markedly from all others implies that $B R A F$ mutation does not simply substitute for KRAS activation in a linear signalling pathway but probably confers additional or distinct properties, with ominous consequences. In cultured cells, the V600E mutation increases BRAF activity independent of KRAS and shows lower transforming activity than oncogenic KRAS (Davies et al, 2002). Likewise, smallmolecule inhibition of MEK abrogated tumour growth more profoundly in BRAF-mutant than KRAS-mutant tumour xenografts (Solit et al, 2006). Such differences may in part underlie the 
contrast in predictive value of activating $K R A S$ and $B R A F$ mutations. Second, the frequency of $B R A F$ mutation is especially high, up to $50 \%$, in tumours with microsatellite instability (MSI ${ }^{\text {hi }}$ ) (Rajagopalan et al, 2002), which otherwise carry a favourable prognosis (Popat et al, 2005). If the outcome of patients with $B R A F$ mutation is so poor, then the natural history of MSI ${ }^{\text {hi }}$ tumours lacking $B R A F$ mutation must be even more favourable; a recent study of patients with operable stage II or stage III CRC made the same suggestion (French et al, 2008). If this idea is confirmed, patients with such tumors may, in the future, be candidates for prolonged observation without treatment.

In agreement with recent reports (Bokemeyer et al, 2008; Van Cutsem et al, 2009), mutant or WT status for KRAS did not correlate with the outcomes in patients treated with front-line 5FU-based regimens. Although a previous study reported worse outcomes in patients with early (stages II-III) PIK3CA-mutant CRC (Kato et al, 2007), the aggregate data do not currently suggest that KRAS- or PIK3CA-mutant tumours have distinctive clinical profiles or differential response to currently approved chemotherapy. The lack of overall predictive significance of KRAS mutation despite its effect on cetuximab treatment hints that the adverse effect of BRAF mutation is not explained just by the limited response to cetuximab.

This study reinforces the value of mutational analysis in patients treated with EGFR mAb. Patients with KRAS-mutant tumours treated with cetuximab in any combination had lower PFS compared with those with KRAS ${ }^{\mathrm{WT}}$ tumours, results that extend those reported in recent clinical trials (Bokemeyer et al, 2008; Van Cutsem et al, 2009) and suggest that KRAS mutation may bypass aberrant EGFR signalling. We observed similar trends in the likelihood of response between patients with BRAF-mutant or $B R A F^{\mathrm{WT}}$ tumours $(0 \mathrm{vs} 17 \%)$. Although responses in PIK3CAmutant cases were not clearly as worse as they were in cases with KRAS or BRAF mutation, PFS with cetuximab-based therapy was significantly lower when tumors carried mutations in any of the three examined genes, and both $B R A F$ (HR 3.9, $P=0.0005)$ and PIK3CA (HR 2.1, $P=0.01$ ) mutations conferred higher risk of relapse after treatment with cetuximab-containing salvage combinations. Two studies that previously considered the significance of $B R A F$ and PIK3CA mutations in treatment response to anti-EGFR $\mathrm{mAb}$ (Moroni et al, 2005; Lievre et al, 2006) had small sample sizes (30 and 31 cases, fewer than three with mutations). Our study extends the current paradigm to imply that patients with KRAS-, $B R A F$ - or PIK3CA-mutant tumours may all derive limited benefit from treatment with EGFR $\mathrm{mAb}$, and that BRAF mutations in particular account for a measurable fraction of patients whose KRAS ${ }^{W T}$ tumours do not respond to cetuximab. Reduced PFS in PIK3CA-mutant mCRC is not simply a function of poorer prognosis because overall outcomes are unrelated to this genetic event (Figure 1). Indeed, KRAS and PIK3CA mutations were not associated with worse OS, possibly because $82 \%$ of patients received another regimen after progression on cetuximabbased treatment or because response to cetuximab impacts modestly on the natural history of mCRC. (Messersmith and Ahnen, 2008).

Colorectal tumours are typically accessible for molecular characterization and common mutations localize in specific exons. Although it is formally possible that the mutational profile of metastatic deposits differs from that in the primary tumour, recent analyses indicate that this is not the case, (Jones et al, 2008); in any event, our analysis shows certain molecular lesions in the primary tumor as markers of the clinical outcome in mCRC. As we studied a large number of unselected cases with good representation of index mutations, the results are robust and directly relevant to current management of mCRC. They advance application of CRC mutation profiles to define patient sub-groups and should influence patient selection or stratification for prospective trials. KRAS and PIK3CA mutations seem not to be useful predictors for response to current treatment regimens. If our conclusions are confirmed independently, then patients with the BRAF V600E mutation might justify foregoing approved treatments in favour of investigational therapy. It will also be instructive to confirm the idea that like KRAS mutations, those in $B R A F$ and PIK3CA also predict for lack of clinical benefit from EGFR mAb therapy.

\section{ACKNOWLEDGEMENTS}

We thank Laura MacConnaill and Levi Garraway for assistance with tumour genotyping and Dr Robert Mayer for critical reading of the paper. The study was supported by the Dana-Farber Cancer Institute-Novartis Drug Discovery Program (RAS), SPORE grant P50CA127003 from the National Institutes of Health and a research fellowship from the Hellenic Society of Medical Oncology (JS).

Supplementary Information accompanies the paper on British Journal of Cancer website (http://www.nature.com/bjc)

\section{REFERENCES}

Amado RG, Wolf M, Peeters M, Van CE, Siena S, Freeman DJ, Juan T, Sikorski R, Suggs S, Radinsky R, Patterson SD, Chang DD (2008) Wildtype KRAS is required for panitumumab efficacy in patients with metastatic colorectal cancer. J Clin Oncol 26: 1626-1634

Bokemeyer C, Bondarenko I, Makhson A, Hartmann JT, Aparicio J, de BF, Donea S, Ludwig H, Schuch G, Stroh C, Loos AH, Zubel A, Koralewski P (2008) Fluorouracil, leucovorin, and oxaliplatin with and without cetuximab in the first-line treatment of metastatic colorectal cancer. J Clin Oncol 27: 663-671

Cunningham D, Humblet Y, Siena S, Khayat D, Bleiberg H, Santoro A, Bets D, Mueser M, Harstrick A, Verslype C, Chau I, Van CE (2004) Cetuximab monotherapy and cetuximab plus irinotecan in irinotecan-refractory metastatic colorectal cancer. $N$ Engl J Med 351: 337-345

Davies H, Bignell GR, Cox C, Stephens P, Edkins S, Clegg S, Teague J, Woffendin H, Garnett MJ, Bottomley W, Davis N, Dicks E, Ewing R, Floyd Y, Gray K, Hall S, Hawes R, Hughes J, Kosmidou V, Menzies A, Mould C, Parker A, Stevens C, Watt S, Hooper S, Wilson R, Jayatilake H, Gusterson BA, Cooper C, Shipley J, Hargrave D, Pritchard-Jones K, Maitland N, Chenevix-Trench G, Riggins GJ, Bigner DD, Palmieri G, Cossu A, Flanagan A, Nicholson A, Ho JW, Leung SY, Yuen ST, Weber

BL, Seigler HF, Darrow TL, Paterson H, Marais R, Marshall CJ, Wooster $R$, Stratton MR, Futreal PA (2002) Mutations of the BRAF gene in human cancer. Nature 417: 949-954

French AJ, Sargent DJ, Burgart LJ, Foster NR, Kabat BF, Goldberg R, Shepherd L, Windschitl HE, Thibodeau SN (2008) Prognostic significance of defective mismatch repair and BRAF V600E in patients with colon cancer. Clin Cancer Res 14: 3408-3415

Grothey A, Sargent D, Goldberg RM, Schmoll HJ (2004) Survival of patients with advanced colorectal cancer improves with the availability of fluorouracil-leucovorin, irinotecan, and oxaliplatin in the course of treatment. J Clin Oncol 22: 1209-1214

Huang CH, Mandelker D, Schmidt-Kittler O, Samuels Y, Velculescu VE, Kinzler KW, Vogelstein B, Gabelli SB, Amzel LM (2007) The structure of a human p110alpha/p85alpha complex elucidates the effects of oncogenic PI3Kalpha mutations. Science 318: 1744-1748

Hurwitz H, Fehrenbacher L, Novotny W, Cartwright T, Hainsworth J, Heim W, Berlin J, Baron A, Griffing S, Holmgren E, Ferrara N, Fyfe G, Rogers B, Ross R, Kabbinavar F (2004) Bevacizumab plus irinotecan, fluorouracil, and leucovorin for metastatic colorectal cancer. $N$ Engl J Med 350: 2335-2342 
Jones S, Chen WD, Parmigiani G, Diehl F, Beerenwinkel N, Antal T, Traulsen A, Nowak MA, Siegel C, Velculescu VE, Kinzler KW, Vogelstein B, Willis J, Markowitz SD (2008) Comparative lesion sequencing provides insights into tumor evolution. Proc Natl Acad Sci USA 105: $4283-4288$

Karapetis CS, Khambata-Ford S, Jonker DJ, O'Callaghan CJ, Tu D, Tebbutt NC, Simes RJ, Chalchal H, Shapiro JD, Robitaille S, Price TJ, Shepherd L, Au HJ, Langer C, Moore MJ, Zalcberg JR (2008) K-ras mutations and benefit from cetuximab in advanced colorectal cancer. $N$ Engl J Med 359: $1757-1765$

Kato S, Iida S, Higuchi T, Ishikawa T, Takagi Y, Yasuno M, Enomoto M, Uetake H, Sugihara K (2007) PIK3CA mutation is predictive of poor survival in patients with colorectal cancer. Int J Cancer 121: 1771-1778

Lievre A, Bachet JB, Boige V, Cayre A, Le CD, Buc E, Ychou M, Bouche O, Landi B, Louvet C, Andre T, Bibeau F, Diebold MD, Rougier P, Ducreux M, Tomasic G, Emile JF, Penault-Llorca F, Laurent-Puig P (2008) KRAS mutations as an independent prognostic factor in patients with advanced colorectal cancer treated with cetuximab. J Clin Oncol 26: 374-379

Lievre A, Bachet JB, Le CD, Boige V, Landi B, Emile JF, Cote JF, Tomasic G, Penna C, Ducreux M, Rougier P, Penault-Llorca F, Laurent-Puig P (2006) KRAS mutation status is predictive of response to cetuximab therapy in colorectal cancer. Cancer Res 66: 3992-3995

Messersmith WA, Ahnen DJ (2008) Targeting EGFR in colorectal cancer. $N$ Engl J Med 359: 1834-1836

Moroni M, Veronese S, Benvenuti S, Marrapese G, Sartore-Bianchi A, Di NF, Gambacorta M, Siena S, Bardelli A (2005) Gene copy number for epidermal growth factor receptor (EGFR) and clinical response to antiEGFR treatment in colorectal cancer: a cohort study. Lancet Oncol 6: $279-286$

Popat S, Hubner R, Houlston RS (2005) Systematic review of microsatellite instability and colorectal cancer prognosis. J Clin Oncol 23: 609-618

Rajagopalan H, Bardelli A, Lengauer C, Kinzler KW, Vogelstein B, Velculescu VE (2002) Tumorigenesis: RAF/RAS oncogenes and mismatch-repair status. Nature 418: 934

Samowitz WS, Sweeney C, Herrick J, Albertsen H, Levin TR, Murtaugh MA, Wolff RK, Slattery ML (2005) Poor survival associated with the BRAF V600E mutation in microsatellite-stable colon cancers. Cancer Res 65: $6063-6069$

Schubbert S, Shannon K, Bollag G (2007) Hyperactive Ras in developmental disorders and cancer. Nat Rev Cancer 7: 295-308
Solit DB, Garraway LA, Pratilas CA, Sawai A, Getz G, Basso A, Ye Q, Lobo JM, She Y, Osman I, Golub TR, Sebolt-Leopold J, Sellers WR, Rosen N (2006) BRAF mutation predicts sensitivity to MEK inhibition. Nature 439: $358-362$

Thomas RK, Baker AC, Debiasi RM, Winckler W, Laframboise T, Lin WM, Wang M, Feng W, Zander T, MacConaill L, Lee JC, Nicoletti R, Hatton C, Goyette M, Girard L, Majmudar K, Ziaugra L, Wong KK, Gabriel S, Beroukhim R, Peyton M, Barretina J, Dutt A, Emery C, Greulich H, Shah K, Sasaki H, Gazdar A, Minna J, Armstrong SA, Mellinghoff IK, Hodi FS, Dranoff G, Mischel PS, Cloughesy TF, Nelson SF, Liau LM, Mertz K, Rubin MA, Moch H, Loda M, Catalona W, Fletcher J, Signoretti S, Kaye F, Anderson KC, Demetri GD, Dummer R, Wagner S, Herlyn M, Sellers WR, Meyerson M, Garraway LA (2007) High-throughput oncogene mutation profiling in human cancer. Nat Genet 39: 347-351

Van Cutsem E, Lang I, D'haens I, Moiseyenko V, Zaluski J, Folprecht G, Tejpar S, Kisker O, Stroh C, Rougier P (2009) KRAS status and efficacy in the first-line treatment of patients with metastatic colorectal cancer (mCRC) treated with FOLFIRI with or without cetuximab: The CRYSTAL experience. J Clin Oncol 26, Ref Type: Abstract

Van CE, Peeters M, Siena S, Humblet Y, Hendlisz A, Neyns B, Canon JL, Van Laethem JL, Maurel J, Richardson G, Wolf M, Amado RG (2007) Open-label phase III trial of panitumumab plus best supportive care compared with best supportive care alone in patients with chemotherapy-refractory metastatic colorectal cancer. J Clin Oncol 25: $1658-1664$

Vogelstein B, Kinzler KW (2004) Cancer genes and the pathways they control. Nat Med 10: 789-799

Wood LD, Parsons DW, Jones S, Lin J, Sjoblom T, Leary RJ, Shen D, Boca SM, Barber T, Ptak J, Silliman N, Szabo S, Dezso Z, Ustyanksky V, Nikolskaya T, Nikolsky Y, Karchin R, Wilson PA, Kaminker JS, Zhang Z, Croshaw R, Willis J, Dawson D, Shipitsin M, Willson JK, Sukumar S, Polyak K, Park BH, Pethiyagoda CL, Pant PV, Ballinger DG, Sparks AB, Hartigan J, Smith DR, Suh E, Papadopoulos N, Buckhaults P, Markowitz SD, Parmigiani G, Kinzler KW, Velculescu VE, Vogelstein B (2007) The genomic landscapes of human breast and colorectal cancers. Science 318: $1108-1113$

Yuen ST, Davies H, Chan TL, Ho JW, Bignell GR, Cox C, Stephens P, Edkins S, Tsui WW, Chan AS, Futreal PA, Stratton MR, Wooster R, Leung SY (2002) Similarity of the phenotypic patterns associated with BRAF and KRAS mutations in colorectal neoplasia. Cancer Res 62: 6451-6455 